\title{
Immune resistance and tolerance strategies in malaria vector and non-vector mosquitoes
}

Tibebu Habtewold ${ }^{1,2^{*}}$, Zoe Groom ${ }^{1,3}$ and George K. Christophides ${ }^{1}$

\begin{abstract}
Background: The Anopheles gambiae complex consists of species that vary greatly in their capacity to transmit malaria. The mosquito immune system has been identified as a key factor that can influence whether Plasmodium infection establishes within the mosquito vector. This study was designed to investigate the immune responses of An. coluzzii, An. arabiensis and An. quadriannulatus mosquitoes. The first two mosquito species are major vectors of malaria in sub-Saharan Africa, while the third is thought to be a non-vector.
\end{abstract}

Methods: All three mosquito species were reared in mixed cultures. Their capacity to eliminate $P$. berghei and regulate midgut bacteria was examined.

Results: Our results revealed large differences in mosquito resistance to $P$. berghei. In all three mosquito species, immune reactions involving the complement system were triggered when the number of parasites that mosquitoes were challenged with exceeded a certain level, i.e. immune tolerance threshold. This threshold was markedly lower in An. quadriannulatus compared to An. coluzzii and An. arabiensis. We also demonstrated that the level of immune tolerance to $P$. berghei infection in the haemolymph is inversely correlated with the level of immune tolerance to microbiota observed in the midgut lumen after a blood meal. The malaria non-vector mosquito species, An. quadriannulatus was shown to have a much higher level of tolerance to microbiota in the midgut than An. coluzzii.

Conclusions: We propose a model whereby an increased tolerance to microbiota in the mosquito midgut results in lower tolerance to Plasmodium infection. In this model, malaria non-vector mosquito species are expected to have increased immune resistance in the haemocoel, possibly due to complement priming by microbiota elicitors. We propose that this strategy is employed by the malaria non-vector mosquito, An. quadriannulatus, while An. coluzzii has reduced tolerance to bacterial infection in the midgut and consequently reduced immune resistance to Plasmodium infection at the haemocoel level. An in-depth understanding of the molecular mechanisms regulating immune tolerance versus resistance in different mosquito vectors of malaria could guide the design of new vector and disease control strategies.

Keywords: Mosquito immunity, Pathogen tolerance/resistance, Plasmodium, Microbiota, Haemolymph antimicrobial activity

\footnotetext{
* Correspondence: t.habtewold@imperial.ac.uk

${ }^{1}$ Department of Life Sciences, Imperial College London, London, UK

${ }^{2}$ Department of Comparative Physiology and Biometrics, University of Ghent,

Ghent, Belgium

Full list of author information is available at the end of the article
} 


\section{Background}

The mosquito immune system has been identified as a key factor that can influence whether Plasmodium infection establishes within the mosquito vector and is the parasite is therefore transmitted to another host. Parasites encounter robust immune responses while invading and developing in the mosquito midgut, which often leads to their elimination. Parasite killing primarily takes place in two major mosquito compartments: the midgut lumen and the haemocoel. Immune responses in the mosquito midgut mainly involve the immune deficiency (Imd) pathway and the $N F-\kappa B$ transcription factor; REL2 [1]. The Imd pathway is responsible for maintaining a basal level of immune activity, and its activation relies mostly on recognition of peptidoglycan, a cell wall component found in almost all bacteria. In mosquitoes, and also fruit flies, peptidoglycan shed by the gut microbiota is recognised by peptidoglycan recognition protein LC (PGRPLC). This leads to nuclear translocation of REL2 and production of immune effector molecules [2]. Following a blood meal, bacterial levels increase with a consequent increase of peptidoglycan that enhances the responses of the Imd pathway [3]. Immune effectors of the Imd pathway, synthesised in response to gut bacteria, increase and kill Plasmodium parasites that may be present following the ingestion of a blood meal [2]. Additional receptors involved in immune responses to midgut enterobacteria have been revealed, but the signalling pathways through which they function remain unknown [4]. The receptors include three fibronectin type III domain proteins, two of which contain immunoglobulin domains, and a gustatory receptor. The gustatory receptor has been shown to control satiation to sugar meals, providing a link between immune and behavioural responses.

In the haemocoel, immune responses involve humoral reactions of the haemolymph, primarily the complementlike pathway. The Anopheles thioester-containing protein 1 (TEP1), together with two leucine-rich repeat proteins; LRIM1 and APL1, and several CLIP-domain serine protease homologs, play a principal role in the complementlike pathway [5]. TEP1 and CLIPs bind to the surface of Plasmodium ookinetes as soon as they appear on the basal sub-epithelial space of the midgut, marking them for lysis and, in some mosquito strains, melanisation $[6,7]$. It has been shown that the TEP1 gene is highly polymorphic in wild An. gambiae and An. coluzzii populations [8] and that there is an association between certain TEP1 alleles and resistance to Plasmodium [9, 10]. Complement-like pathway responses can also be triggered by bacteria that may be introduced into the hemolymph either artificially [5] or through breaches of the gut epithelium such as those caused by the invasion of malaria parasites [11]. Basal levels of TEP1, and presumably other components of this pathway, are also controlled by a signalling pathway orthologous to Drosophila Toll that involves the $N F-\kappa B$ transcription factor, REL1 [12]. Increased pre-infection levels of TEP1 have been observed following knockdown of the inhibitor of REL1 nuclear translocation; Cactus [13]. For instance, activation of the Toll pathway in $A n$. gambiae mosquitoes through the silencing of Cactus have been shown to result in increased $P$. berghie killing by TEP1 [13]. Previous publications have shown that Cactus is a negative regulator of Toll (REL1), which in turn is the upstream regulator of the complement-like pathway [14].

Mosquito hosts respond to pathogen challenges through a combination of immune resistance and tolerance mechanisms. A balance between these two types of response ensures protection from pathogens and, at the same time, limits the collateral damage that may arise as a result of excessive immune effector production. Immune resistance comprises of multiple mechanisms that either prevent pathogens from invading or eliminate pathogens after the invasion has taken place. Immune tolerance mechanisms primarily involve lowered responsiveness to particular immune stimuli and the initiation of processes that protect host tissues from damage caused by the pathogen. This often depends upon the intensity of the challenge by the pathogen. Examples include up-regulation of wound healing and down-regulation of immunopathology, which is often associated with collateral tissue damage, or mitigation of pathogen virulence, through the up-regulation of proteins that can protect host tissues from endotoxins [15-17]. Some molecules have been identified in Drosophila, that have been shown to promote tolerance to gut bacteria through the inhibition of the Imd pathway [18-20]. In mosquito, for instance, the DuoxIMPer (Dual oxidase - Immunomodulatory peroxidase) module is induced after blood-feeding. This facilitates the formation of a dityrosine-linked mucus layer that plugs the ectoperitrophic space (space between the peritrophic membrane and the midgut epithelium) leading to reduced permeability to microbial antigens [21]. As with resistance, mosquito tolerance is believed to be partly regulated by gut microbiota through specialised immune modules, for example, extracellular polysaccharides (EPS). Such molecules block host immune signalling [22-26]. The effect of this blockade varies in magnitude between resistant and susceptible hosts.

Malaria parasites are believed to exploit the interplay between immune tolerance and resistance to establish an infection. Both processes require allocation of resources at the expense of other physiological functions such as survival and reproduction. Mosquito survival and longevity is a key contributing factor to the vectorial capacity of Anopheles. This is because only mosquitoes in which the parasite has developed into the sporozoite stage can transmit malaria [27]. 
We investigate how the balance between immune tolerance and resistance in malaria vector and non-vector species of the An. gambiae complex controls Plasmodium infection. We reveal that the immune responses in the two main mosquito immune compartments, the midgut and the haemocoel, are inversely related. For example, the level of immune response (tolerance/resistance) in the midgut, often against microbiota, appears to regulate the level of immune response in the haemolymph, possibly due to priming of complement reactions. Malaria nonvector mosquito species show a higher tolerance to gut microbiota, resulting in a greater refractoriness to Plasmodium infection, while the opposite is true for malaria vector mosquitoes. The molecular mechanisms regulating immune tolerance versus resistance in different mosquito vectors of malaria could guide the design of new vector and disease control strategies.

\section{Methods}

\section{Mosquito strain}

Anopheles arabiensis DONGOLA strain (Sudan, provided by MR4), An. coluzzii N'gousso strain (originally An. gambiae $\mathrm{M}$ form, colonised from field mosquitoes in Yaoundé, Cameroon) and An. quadriannulatus SANGQUA strain (Zimbabwe, provided by MR4) were used throughout the experiments.

\section{Mosquito rearing and maintenance}

Mosquitoes were reared and maintained as previously described [28], separately or in mixed cultures. In mixed cultures, equal numbers of L1 stage larvae from each species were pooled together. The individual mosquito species were identified during, or at the end of experiments, using PCR-based methods as previously described [29].

\section{Infection}

A GFP-expressing P. berghei ANKA 2.34 strain [28] was used to infect mosquitoes by direct feeding on gametocytemic mice (at 7-8\% parasitaemia) as previously described [28] or through the use of membrane feeders using an overnight ookinete culture. The ookinete cultures were first pelleted, and the pellet was then suspended in human blood serum at concentrations of $10^{4}$, $10^{5}, 10^{6}$, and $10^{7}$ ookinetes $/ \mathrm{ml}$. The average blood meal volume of mosquitoes within the An. gambiae complex is about $2 \mu \mathrm{l}$ (unpublished data); hence each mosquito was infected with approximately 20, 200, 2,000 and 20,000 ookinetes, for the respective dilution. This dose range covers optimum ookinetes to oocyst conversion dose previously reported by [30], i.e. 500 to 3000 ookinete/mosquito.

The parasite infection intensity was determined through direct examination of fluorescent oocysts in the midguts [28] or by quantitative real-time PCR (qPCR). The abundance of the CSP gene fragment was measured five days post infection, hence served as a marker for viable oocyst development in the gut.

A modified protocol from [31] was used for Staphylococcus aureus culturing and administration for mosquito infection. Briefly, the bacteria culture was allowed to grow to $\mathrm{OD}_{600}=0.7$ in LB liquid media and then precipitated and washed before suspending in PBS to a final bacterial suspension of $\mathrm{OD}_{600}=0.4$. Bacteria were injected into the mosquito thorax.

\section{Midgut dissection}

Three types of midgut dissection were carried out: (i) Midguts were removed from female mosquitoes that were infected with $P$. berghei 7 days earlier, and the parasite infection intensity in the gut was quantified by direct examination for fluorescent oocysts as previously described [28]. (ii) Midguts infected with ookinetes using membrane feeder were dissected on day five after infection with $P$. berghei. The infection intensity was determined by measuring the abundance of $P$. berghei CSP using qPCR on DNA extracted from the midguts. The midgut dissection was carried out on ice cold PBS, and ten midguts were pooled and then transferred into $1.5 \mathrm{ml}$ Eppendorf tubes with tissue lysis buffer and kept on the ice until DNA processing. DNA processing was carried out within two hours of dissection. (iii) For molecular quantification of midgut microbiota, midguts were removed $24 \mathrm{~h}$ post-blood meal. Before dissection, the mosquitoes were dipped in sterile water. Forceps used for dissection were sterilised in a flame between each dissection. The dissection was carried out using cold PBS on ice and midguts were then immediately transferred to Eppendorf tubes with tissue lysis buffer.

In each of the above experiments, mosquito carcases were used for species ID.

\section{dsRNA synthesis and mosquito injection}

dsRNA production was performed [28] using gene specific oligonucleotide primers flanked by the short T7 promoter sequence TAA TAC GAC TCA CTA TAG GG. Oligonucleotide primer sequences are shown in Additional file 1: Table S1. For each of the genes, the target region was cloned from a cDNA library, and the dsRNA was synthesised using the MEGAscript T7 Kit (Ambion, Huntington, UK) treated with DNAse I and cleaned using the RNeasy kit (Qiagen, Hilden, Germany). dsRNA concentrations were adjusted to $3 \mu \mathrm{g} / \mu \mathrm{l}$. For each dsRNA treatment, 60 female mosquitoes, of two to 3 days old, were placed in a paper cup and injection was performed as previously described [6]. Each mosquito received a volume of $69 \mathrm{ml}$ dsRNA solution injected into the 
lateral side of the thorax. After 3 days, the mosquitoes were challenged with Plasmodium parasite.

Gene silencing experiments using dsRNA were replicated two to three times at an interval of one week or more.

\section{RT-qPCR analysis}

For assessing knockdown efficiency, we measured the level of expression of target genes as described previously [28]. Total RNA was extracted from approximately ten adult mosquitoes using TRIzol reagent (Invitrogen, CA, USA) as described by the protocol. About $1 \mu \mathrm{g}$ of total RNA (the extraction product) was used for reverse transcription using Superscript II (Invitrogen), which was used in the PCR reaction. For measuring the load of P. berghei or microbiota, genomic DNA (gDNA) was extracted from ten pooled midguts using the DNeasy Blood \& Tissue kit (Qiagen, Hilden, Germany), and the extraction product was used as a PCR template. The abundance of $P$. berghei CSP and the bacterial 16S rRNA genes were used to detect parasite infection intensity and midgut bacterial load, respectively.

The total volume of reagents was $20 \mu \mathrm{l}$ containing; $2 \mu \mathrm{l}$ gDNA or cDNA, $10 \mu \mathrm{l}$ of $2 \times \mathrm{SYBR}^{\circ}$ premix Ex Taq (Takara, Maountain view, USA), $0.2 \mu \mathrm{M}$ of each primer and $0.4 \mu \mathrm{l}$ Rox reference dye (50x). Amplification and detection of fluorescence signals were carried out using an Applied Biosystems 7500 Fast Real-Time PCR system. The PCR cycling program consisted of an initial denaturation stage at $95{ }^{\circ} \mathrm{C}$ for $20 \mathrm{~s}$, followed by 40 cycles at $95{ }^{\circ} \mathrm{C}$ for $3 \mathrm{~s}$ and $60{ }^{\circ} \mathrm{C}$ for $30 \mathrm{~s}$. Each gene was quantified in duplicate, and the threshold crossing values $\left(C_{T}\right.$ -values) were standardised (using a standard curve) and normalised to the geometric mean of the S7 rRNA gene that served as an internal control [28].

\section{Detecting microbiota family}

Midgut microbiota families were identified $24 \mathrm{~h}$ postblood meal in each of the three aforementioned mosquito species that were reared in a mixed culture. After dissection, midguts were transferred into separately labelled tubes and kept on ice until the species of mosquito was identified using the PCR method described above. Ten midguts of the same species were pooled, and genomic DNA was extracted using the Wizard ${ }^{\circ}$ Genomic DNA Purification Kit (Promega, Madison, USA), according to manufacturer's instructions. Purified gDNA was resuspended in $50 \mu \mathrm{l}$ of sterile water before PCR amplification. 16S rRNA gene specific primers, $1492 \mathrm{r}$ and $27 \mathrm{f}$ as described in [32] were used to amplify partial sequences ( $>750 \mathrm{bp})$ of a $16 \mathrm{~S}$ ribosomal gene from bacterial isolates. Products were cloned into the PCR-Blunt Vector and used to transform DH5-alpha cells (Invitrogen). For each mosquito species, 100 white colonies were selected randomly, and the $16 \mathrm{~S}$ PCR product was sequenced. ClustalW and NCBI blast software was used to identify the bacterial family from the 16S rRNA sequences.

\section{Haemolymph collection and antimicrobial activity assays} For this experiment, each mosquito species was reared separately as larvae, and the resulting adults were maintained in separate cages. Antimicrobial activity was assessed using a turbidimetry assay in which lytic activity in the haemolymph is determined by the total change in optical density of the aqueous suspension of lyophilized cell wall from Micrococcus luteus (Micrococcus ysodeikticus ATCC No. 4698, Sigma-Aldrich, Saint Louis, USA). The test was performed as described before [33] with some modifications. First, female mosquitoes were starved for $3 \mathrm{~h}$ and were then offered $0.2 \%$ phenylthiourea (PTU) in sugar solution. The mosquitoes were then decapitated and immediately transferred to ice cold Millipore Ultrafree ${ }^{\circ}$-MC Centrifugal Filter Units of $0.22 \mu \mathrm{m}$, containing $60 \mu \mathrm{l} \mathrm{PBS}$ and $0.2 \% \mathrm{PTU}$, $\mathrm{pH}$ 6.4. The tubes were then spun at $13,000 \times \mathrm{rpm}$ for $30 \mathrm{~s}$, and $20 \mu \mathrm{l}$ of the hemolymph collection was pipetted into 96-well plates and kept on ice. Lysozyme solution $(1 \mu \mathrm{g} / \mathrm{ml})$ was included as a control and samples were run in triplicate. A $180 \mu \mathrm{l}$ suspension of M. luteus in PBS $(0.5 \mathrm{mg} / \mathrm{ml})$ was added to each well. $450 \mathrm{~nm}$ absorbance of the substrate-hemolymph mixture was monitored every minute for 60 min using an FLUOstar Omega plate reader. Wells with PBS only were included to control for the decline in turbidity due to settling. The mean change in absorbance of blank samples was subtracted from each experimental reading and the mean change in the $\Delta A_{450}$ absorbance/minute between mosquito species was compared using an analysis of covariance (ANCOVA).

\section{Mosquito survival assays}

Mosquito daily survival was monitored for 14 days. Survival curves were compared using Log-rank and Gehan-BreslowWilcoxon tests. An antibiotic cocktail was used in these assays and included $2 \mathrm{mg} / \mathrm{ml}$ Gentamycin (Sigma-Aldrich) and $2 \mathrm{mg} / \mathrm{ml}$ Ciprofloxacin (Fluka, Sigma-Aldrich).

\section{Statistical analysis}

Oocyst count data were positively skewed. Thus the Mann-Whitney test was used to compare infection intensity distribution between paired species or gene silenced mosquitoes. Descriptive statistics were calculated for individual replicates. Median and interquartile ranges were calculated and showed a consistent pattern of oocyst distribution between replicates for all three mosquito species. This allowed data to be pooled for statistical analysis. 


\section{Results}

\section{Susceptibility to Plasmodium}

Plasmodium berghei infection intensity and prevalence were comparatively investigated in laboratory populations of An. arabiensis, An. coluzzii and An. quadriannulatus mosquitoes that were cultured together from larval stages and genotyped after dissection. A larval coculturing protocol was used to minimise potential bias arising from variations in mosquito rearing and microbial diversity. The results showed significantly higher oocyst numbers in An. coluzzii compared to An. arabiensis $\left(\mathrm{U}_{(18)}=2935 ; Z=50.1, P<0.001\right)$ or An. quadriannulatus $\left(\mathrm{U}_{(18)}=2170 ; \quad Z=38.2, \quad P<0.001\right) \quad$ (Fig. 1a). In addition, over half of $A n$. arabiensis and An. quadriannulatus midguts had no detectable oocysts whereas oocyst prevalence was close to $85 \%$ in An. coluzzii.

LRIM1, APL1C and TEP1 were silenced in co-cultured mosquitoes through injection of dsRNA designed against sequences of $A n$. coluzzii genes. The infection intensity of $P$. berghei increased drastically in all three mosquito species, and for all three gene knockdowns, compared to LacZ dsRNA injected controls (Fig. 1b). No significant difference was observed in the infection intensities (Mann-Whitney test) or prevalence (Chi-square test) between the three species following LRIM1, APL1C or TEP1 silencing. In our previous publication, we attained a high and comparable level of gene silencing efficiency for LRIM1, APLC1 and TEP1 An. coluzzii and An. quadiannulatus, i.e. $>98 \%$ reduction of the transcripts of the genes [28]. In that publication, we also reported a high degree of sequence similarity between these species for the exonic region of LRIM1, APLC1 and TEP1 between the mosquitoes. Comparison of sequences LRIM1, APLC1 and TEP1 obtained from VectorBase also supports extremely high sequence similarity between these mosquitoes.

We tested the hypothesis that anti-parasitic immune responses in the three mosquito species are differentially triggered when levels of infection exceed a certain threshold. Mosquito species were infected with 10-fold serial dilutions of overnight $P$. berghei ookinete cultures. The abundance of $\mathrm{PbCSP}$ transcripts in mosquito midguts was determined after 5 days. CSP abundance was similar between the three mosquito species at the starting ookinete dose $\left(10^{4}\right.$ ookinetes $\left./ \mathrm{ml}\right)$. At this dose, a mosquito receives approximately 20 ookinetes (assuming that the average normal bloodmeal volume of a mosquito within the An. gambiae complex is about $2 \mu \mathrm{l})$. At the next ookinete dose $\left(10^{5}\right.$ ookinetes $\left./ \mathrm{ml}\right), C S P$ abundance increased unevenly; An. coluzzii had higher CSP abundance than $A n$. arabiensis and $A n$. quadriannulatus (Fig. 1c). No significant differences were detected between $A n$. arabiensis and An. quadriannulatus. When fitted to a logistic distribution model, in all three mosquito populations, the CSP transcript levels increased initially but showed an apparent tendency to level off or

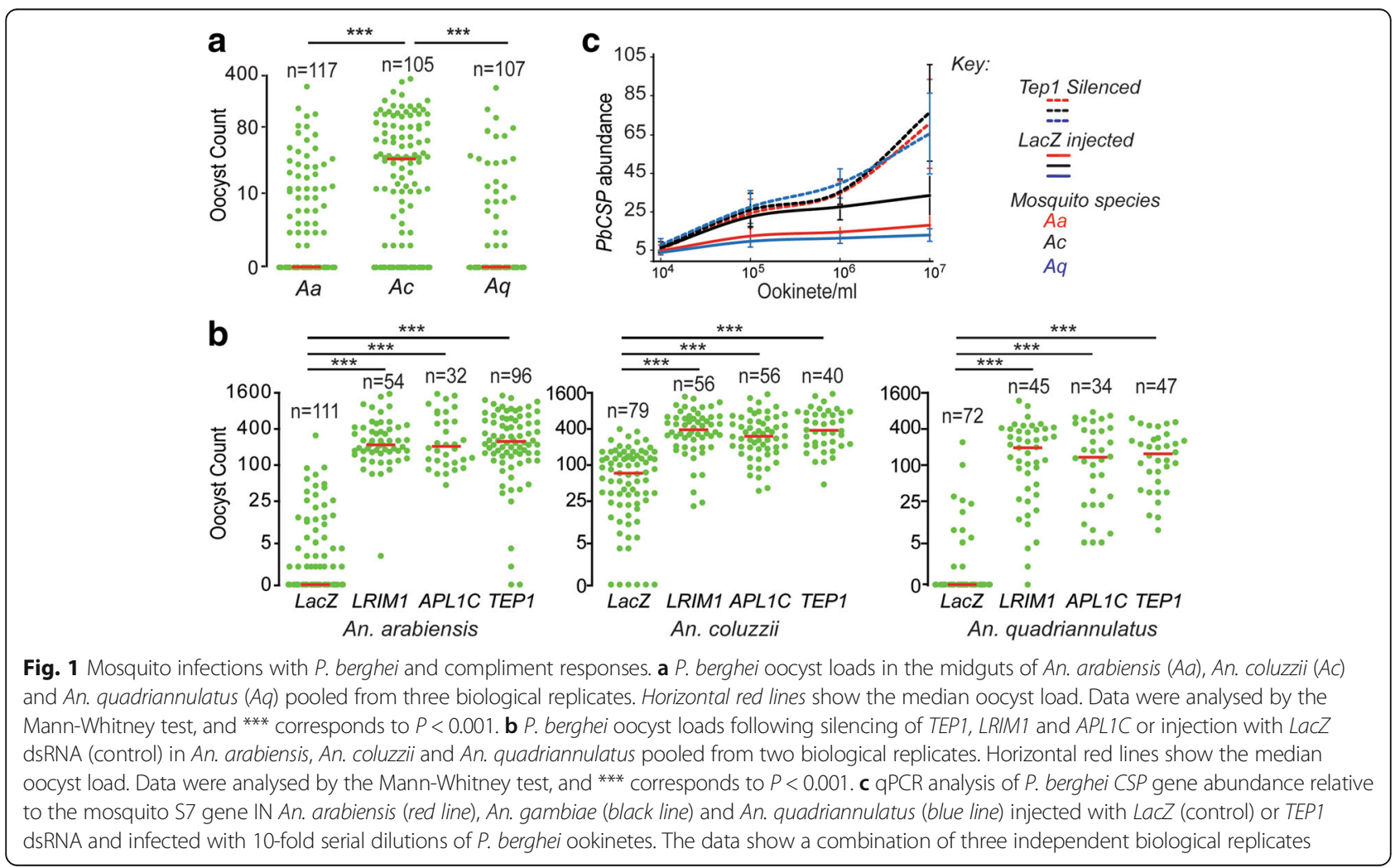


reach a plateau, at higher concentrations $\left(10^{6}\right.$ and $10^{7}$ ookinetes $/ \mathrm{ml}$ ). CSP transcript levels were raised by $5,0.2$ and 0.5 fold in An. arabiensis, 11.7, 0.4, and 0.4 fold in An. coluzzii and 4.5, 0.2 and 0.1 fold following the increase in ookinete dose. Importantly, following TEP1 silencing, CSP transcript levels in all three mosquito species did not increase compared to their respective control mosquitoes at the starting ookinete dose. As the ookinete dose increased, CSP transcript levels also increased equally in all three species. These data indicate that, at low levels of infection, all three mosquito species are equally permissive to parasite infection. We, therefore, hypothesise that no/a weak immune response is directed against parasites at this infection level. Conversely, at higher infection levels, mosquitoes mount strong immune responses against parasites, which are more effective in $A n$. arabiensis and An. quadriannulatus, than in $A n$. coluzzii mosquitoes.

\section{Haemolymph antimicrobial activity}

Previous studies have indicated that haemolymph antimicrobial factors, including antimicrobial peptides and complement factors, are produced in response to immune challenge and that these factors also exhibit anti-Plasmodium activity [34]. We investigated whether heterogeneity in Plasmodium susceptibility between An. arabiensis, An. coluzzii and An. quadriannulatus is due to inherent differences in the robustness of haemolymph antimicrobial activity. Antimicrobial activity of mosquito haemolymph extracts against the gram-positive bacterium Micrococcus luteus was determined using a standard turbidimetric assay. The decline in absorbance was regressed against time and fitted to a model (Fig. 2a). Analysis of covariance (ANCOVA) showed that antimicrobial activity significantly varied among the three mosquito species. When mosquitoes were fed only sugar, significantly higher levels of antimicrobial activity were detected in An. coluzzii than in An. arabiensis $(r=0.98, P<0.001)$ and An. quadriannulatus ( $r=0.99, P<0.001$ ), while An. arabiensis had lower levels of antimicrobial activity compared to An. quadriannulatus $(r=0.98, P<0.001)$. The increased antimicrobial activity in the hemolymph of An. coluzzii may provide a better immune response to pathogens ingested during sugar feeding.

Antimicrobial activity differed substantially between mosquitoes 24-hours post-blood meal compared to the antimicrobial activity observed in sugar-fed mosquitoes. The antimicrobial activity of hemlymph in blood-fed $A n$. coluzzii was significantly reduced compared to sugar-fed An. coluzzii $(r=0.96, P<0.001)$, blood-fed An. arabiensis $(r=0.99, P<0.001)$ and blood-fed An. quadriannulatus $(r=0.98, P<0.001)$. This was in contrast to the antimicrobial activity observed in An. arabiensis $(r=0.96, P$ $<0.001)$ and An. quadriannulatus $(r=0.99, P<0.001)$, both of which increased significantly in blood-fed mosquitoes compared to sugar-fed controls.

To further investigate differences in antimicrobial activity following a bloodmeal, we monitored the abundance of gut microbiota using qPCR. The bacterial $16 \mathrm{~S}$ rRNA gene was used as a quantitative marker of bacterial abundance in co-cultured An. arabiensis, An. coluzzii and An. quadriannulatus. Analysis of qPCR results showed that An. quadriannulatus had a 4-fold higher microbial load than An. arabiensis and a 9-fold higher load than An. coluzzii (Fig. 2b). Microbial numbers were 2 -fold higher in An. arabiensis than in An. coluzzii and this difference were not significant.

Sequencing of 100 16S rRNA PCR products from pools of ten mosquitoes for each of the co-cultured species identified similar microbiota families to those previously
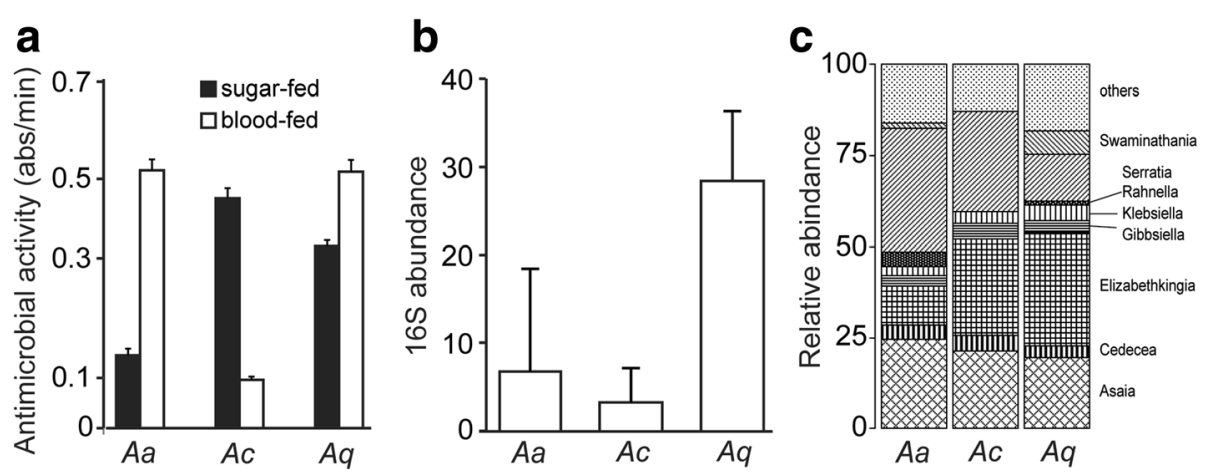

Fig. 2 Hemolymph antimicrobial activity and midgut microbiota load. a Average antimicrobial activity expressed as change in the absorbance of total body fluids of sugar-fed (black bar) and 24-hour blood-fed (white bar) An. arabiensis (Aa), An. coluzzii (Ac) and An. quadriannulatus (Aq) female mosquitoes. Analysis of covariance was used to compare regression lines, $P$-values are shown in the text. Error bars show standard error of the mean for data pooled from 10 midguts in two independent biological replicates. b Median relative abundance of bacterial 16S rRNA gene measured by qPCR. Error bars represent inter-quartile ranges for data from three independent biological experiments, each consisting ten pooled midguts. c Relative abundance of microbiota from the mosquito guts of co-cultured mosquitoes $24 \mathrm{~h}$ after blood-feeding 
reported in the An. coluzzii laboratory colony [35], including Asaia sp., Cedecea sp., Elizabethkingia sp., Gibbsiella sp., Klebsiella sp., Rahnella sp., and Serratia sp. There were no major differences between the three mosquito species for the major microbiota families (Fig. 2c).

\section{Mosquito longevity}

Host immune competence and survival are interrelated and are both important components of host fitness. We monitored the survival rates of mosquito populations to examine the impact of immune responses on fitness. Survival assays were carried out on adult females reared together since initial larval stages using the co-culturing protocol (Fig. 3). Mosquitoes were subject to the following treatments: (a) control, maintained on sugar solution throughout the experiment, (b) blood-fed on naïve mice 3 days post-emergence, and (c) maintained throughout on a sugar solution containing an antibiotic cocktail. A significantly lower survival rate was detected in sugarfed An. arabiensis (48.7\%, $\chi^{2}=23.9, d f=1, P<0.001$ ) and An. quadriannulatus (38.3\%, $\chi^{2}=37.6, d f=1, P<$ 0.001) mosquitoes compared to An. coluzzii (75.8\%) (Fig. 3a). These differences did not change after mosquitoes received a blood meal, i.e. for $A n$. coluzzii (72.9\%) vs An. arabiensis (38.9\%, $\chi^{2}=21.5$, $d f=1, P<$ 0.001); An. coluzzii vs An. quadriannulatus (47.4\%, $\chi^{2}=$ 14.5; $d f=1 ; P<0.001$ ) (Fig. 3b). However, the survival rates of An. quadriannulatus (63.5\%) and An. arabiensis (65.2\%) increased when mosquitoes were treated with antibiotics (Fig. 3c). Survival rates were highest in $A n$. coluzzii $(74.5 \%)$. These data suggest that the high gut microbiota loads in An. quadriannulatus compared to An. coluzzii may negatively impact on mosquito survival.

We investigated the effect of infection on mosquito longevity by monitoring the survival rates of the three species under the following treatments: (d) blood-feeding on $P$. berghei infected mice 3 days post-emergence, and (e) infection (via hemolymph inoculation) with Staphylococcus aureus bacteria (unknown lab strain) 3 days postemergence. The results showed that differences in survival rates between the mosquito species were removed when they were challenged with $P$. berghei or $S$. aureus, respectively (Fig. 3d, e). The survival rates for $P$. berghei challenged An. coluzzii, An. arabiensis and An. quadriannulatus were 65.4, 57.7 and 59.4\%, respectively, and survival rates after $S$. aureus infection were 52.1, 44.0 and
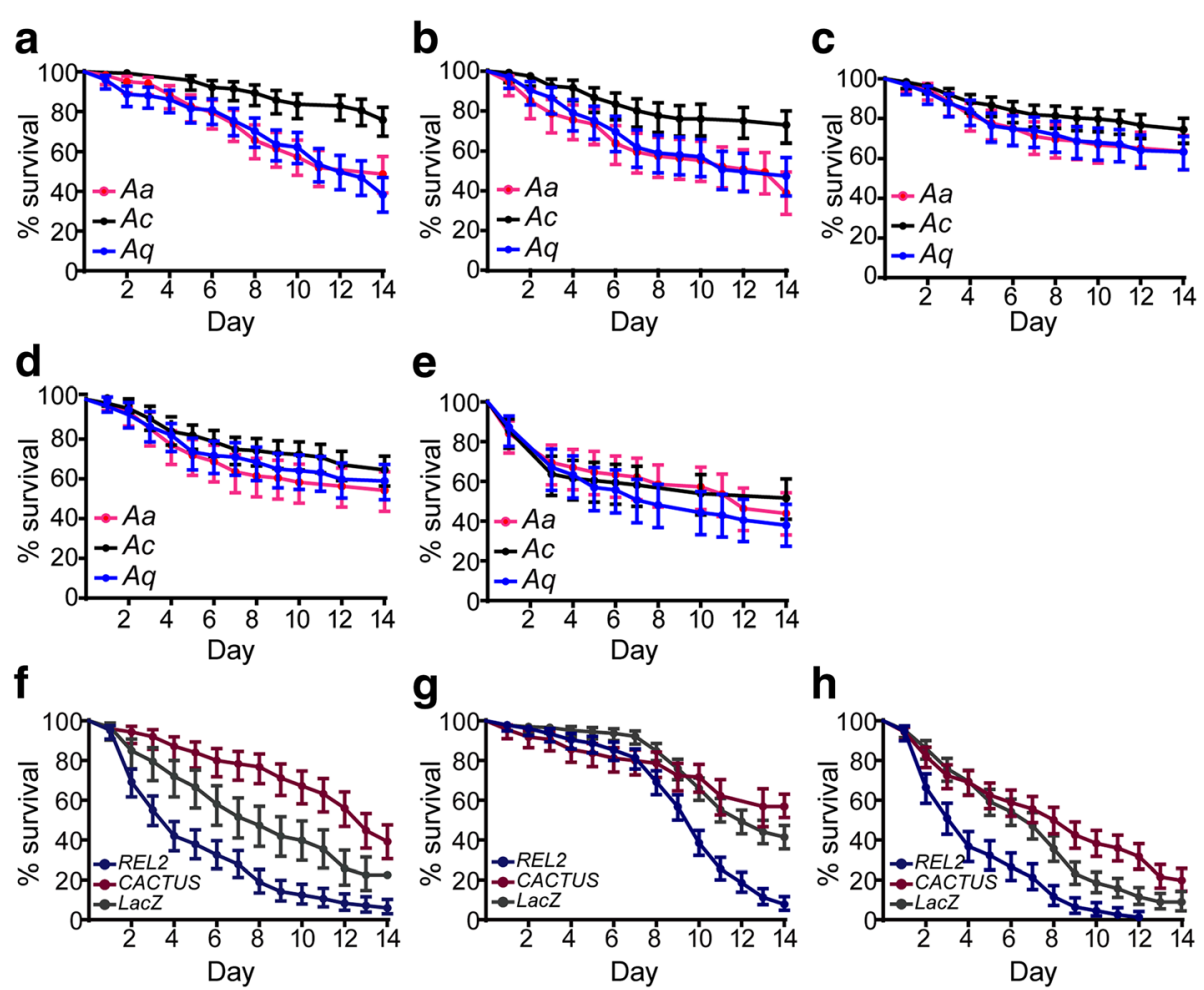

Fig. 3 Daily survival of adult mosquitoes. a-c Survival curves of adult An. arabiensis (Aa), An. coluzzii (Ac) and An. quadriannulatus (Aq) female mosquitoes maintained on sugar solution (a), obtained a single bloodmeal from non-infectious mice on day 3 post-emergence (b) or maintained on sugar solution supplemented with antibiotics for the first 3 days post-emergence (c). Survival curves of adult female mosquitoes infected with P. berghei parasite (d) by direct feeding on gametocytemic mice (at 7-8\% parasitaemia) or injected with S. aureus (e). f-h Survival curves of adult female An. arabiensis (f), An. coluzzii (g) and An. quadriannulatus (h) injected with LacZ (control), REL2 and Cactus dsRNA. Error bars represent 95\% $\mathrm{Cl}$ for three independent biological replicates 
$38.7 \%$. This effect is most likely due to the life-shortening effect of pathogens on An. coluzzii. Taken together, these data suggest that high levels of hemocoel immunity, influenced by an increased abundance of gut microbiota, which is related to reduced epithelial immune response (increased tolerance to bacteria), can be beneficial to mosquitoes during infection but detrimental in the absence of infection. The converse is true for reduced levels of hemocoel immunity (tolerance levels), which impact mosquitoes positively in the absence of infection but negatively during infection.

A significant increase in the proliferation of bacteria has been observed in REL2 silenced An. gambiae mosquitoes, but the microbial level was less when REL1 mediated Cactus expression was silenced. To test the hypothesis that mosquito immune competence and survival are interrelated, we knocked down in co-cultured mosquito populations the $N F-\kappa B$ transcription factor REL2 and thus silenced the antibacterial Imd pathway [36], and the negative regulator of REL1, Cactus [13]. As a result, we ectopically activated the Toll pathway that shares most of its downstream effectors with those of the Imd pathway and is thought to be antifungal (unpublished data). Mosquitoes injected with $d s L a c Z$ served as controls.

A similar and significant silencing efficiency was achieved in all three mosquito species: $60-64 \%$ for REL2 and $78-82 \%$ for Cactus. The level of 16S rRNA gene was not measured in REL2 and Cactus silenced mosquitoes, because we expect similar results as the previously published observations by [2] with these genes/pathways on the bacterial in the midguts. Over the monitoring period, silencing REL2 significantly decreased survival, in An. arabiensis (5.9\%, $\left.X^{2}=26.0, d f=1, P<0.001\right), A n$. coluzzii $\left(7.8 \%, X^{2}=78.9, d f=1, P<0.001\right)$ and $A n$. quadriannulatus $\left(0 \%, X^{2}=44.2, d f=1, P<0.001\right)$ compared to the $L a c Z$ treatments in all three populations (Fig. $3 \mathrm{f}$ h). The survival rate, in the respective mosquito species, was $22.4,41.5$ and $8.9 \%$ for $L a c Z$ treatment. Interestingly, the effect of REL2 silencing on An. coluzzii became apparent only late in mosquito life (Fig. 3g). This confirms the functional importance of the Imd pathway for mosquito survival. LacZ treated group, Cactus silencing significantly increased survival of $A n$. arabiensis (42.8\%) $\left(\chi^{2}=15.3, d f=1, P<0.001\right)$ and An. quadriannulatus $19.8 \%\left(\chi^{2}=13.5, d f=1, P<0.001\right)$ but not $A n$. coluzzii mosquitoes (56.8\%). The survival curves for Cactus and REL2 silenced mosquitoes was significantly different for each mosquito species $\left(\chi^{2}=94.1, d f=1, P\right.$ $<0.001$ for An. arabiensis; $X^{2}=63, d f=1, P<0.001$ for An. coluzzii; and $\chi^{2}=78.2$, $d f=1, P<0.001$ for $A n$. quadriannulatus). These data suggest that compromising the mosquito antibacterial immune response has a deleterious effect on survival, even when mosquitoes are not additionally challenged with a pathogen. Boosting basal levels of immunity before infection provides a fitness advantage to An. arabiensis and An. quadriannulatus but not An. coluzzii.

\section{Discussion}

Differences in vectorial capacity between sibling mosquito species of the An. gambiae complex provides an excellent framework to investigate factors contributing to phenotypes associated with Plasmodium infection and malaria transmission. In this study, experiments were conducted using a mosquito management protocol that minimises possible biases introduced during mosquito rearing, maintenance and handling. Anopheles arabiensis, An. coluzzii and An. quadriannulatus mosquitoes were reared in a mixed culture starting from first-instar larva (L1) and maintained together until completion of the experiments conducted during adulthood.

We confirmed that between the three mosquito species, An. coluzzii is the most permissive to infection with $P$. berghei, while An. quadriannulatus is the most refractory. This is consistent with findings of previous studies that used both P. berghei and P. falciparum [28, 37]. We have also revealed that An. arabiensis is highly refractory to $P$. berghei. This contrasts with the high Victorial Capacity in the $A n$. arabiensis that have been reported across sub-Saharan Africa and also previous observations made in field experiments in West Africa indicating that the mosquito species is as susceptible to $P$. falciparum as An. coluzzii [38]. One of the possible explanations for this discrepancy is that these two sibling African vectors of human malaria have adapted to have increased tolerance to $P$. falciparum as a result of their sympatric co-evolution [39]. In addition, a recent study has revealed that genetic variation within mosquito populations can greatly affect their susceptibility to Plasmodium infection [40].

Our data show that resistance to Plasmodium infection in An. arabiensis and An. quadriannulatus is mediated by the mosquito immune system. Both species become highly and equally susceptible to infection when the expression of key molecules of the complement pathway are silenced. These data support previous findings showing that Plasmodium invasion of the mosquito midgut is largely limited by reactions of the complement pathway in An. coluzzii mosquitoes [6, 28, 41]. Importantly, neither susceptibility nor resistance to parasites was fully penetrant within the three populations. These data, in conjunction with the finding that all three populations demonstrated similar infection phenotypes following complement knockout, suggest the presence of genetic variation associated with the infection phenotype of each population. Indeed, we have been able to genetically select both a highly permissive and a highly 
refractory $A n$. arabiensis line within a limited number of generations (data not shown).

All three mosquito species appear to be equally permissive to $P$. berghei infection at low infection rates. We have shown that oocyst loads in the midguts of An. arabiensis, An. quadriannulatus and An. coluzzii are very similar after challenge with a small number of parasites and are not affected by silencing of the complement pathway. These data suggest that complement reactions are not triggered at low infection rates. Infection intensity dependent responses have been previously reported for $A n$. coluzzii infected with natural populations of $P$. falciparum [42]. Furthermore, reduced differences in susceptibility to $P$. berghei among An. stephensi mosquitoes of a laboratory colony have been previously reported at low infection intensities [43]. Similar observations have been made for Onchocerca roundworm infections of blackflies [44].

Differences in infection intensities between the three mosquito species become significant as the parasite challenge increases. At a dose of approximately 200 ookinetes/mosquito, infection intensities reach a plateau in An. arabiensis and An. quadriannulatus and further dose increase cause little or no change unless the complement system is inactivated. This level of tolerance is ten times higher in An. coluzzii (higher by approximately 2000 ookinetes per mosquito). Previous plant and animal studies have demonstrated the existence of variation in tolerance to pathogenic infections in different breeds, strains or individuals $[45,46]$. Together, our data indicate that differences in susceptibility to parasite infection between the three closely related mosquito species are due to different levels of immune tolerance. The immune responses are activated in all three species as soon as the respective levels of immune tolerance are breached when the pathogen challenge passes a respective threshold.

Basal levels of mosquito immune response are thought to be modulated by midgut microbiota that activates immune signalling pathways leading to the production of antimicrobial effectors [31, 34, 47, 48]. An increase in microbial loads, such as that observed after bloodfeeding, further increases the levels of antimicrobial effectors. These effectors also have anti-parasitic activities. The haemolymph antimicrobial activities of An. quadriannulatus and An. arabiensis $24 \mathrm{~h}$ after bloodfeeding dramatically increases, reaching much higher levels than An. coluzzii. At the same time, their gut microbiota loads were higher than those of An. coluz$z i i$. These data suggest the presence of 2 distinct immune compartments; the midgut lumen and the haemocoel, each with an opposite level of immune tolerance or resistance to the other. Note worth that the haemolymph antimicrobial activity was measured as the rate of digestion of cell wall from grampositive bacterium Micrococcus luteus.

In this present work, midgut microbiota was not measured in the REL2 and Cactus KD An. arabiensis and An. quadriannulatus mosquitoes because we assume that there are no major departures from previously published observations in An. gambiae [2]. The authors demonstrated that the abundance of microbiota increased significantly in REL2 KD An. gambiae mosquitoes compared to $L a c Z$ injected mosquitoes, but there was a significant reduction in the microbial number in Cactus KD mosquitoes.

Our data lead us to propose the following model of immune tolerance versus resistance in malaria vector An. coluzzii non-vector An. quadriannulatus investigated in this study (Fig. 4). The two mosquito species follow distinctly different mechanism of immune response. An. quadriannulatus has elevated levels of immune response at the midgut level compared to $A n$. coluzzii, which leads to high gut microbial load following blood-feeding. These high microbial loads are associated with elevated levels of bacterial-derived immune elicitors such as peptidoglycan that trigger activation of the Imd and possibly other immune pathways, both locally in the gut and systemically in the haemocoel. Immune pathway activation in the gut aims to control bacterial levels and restore homoeostasis, while systemic immune responses in the haemocoel aim to prevent pathogens from breaching the epithelial barrier and establishing infection. The latter leads to elevated levels of resistance against Plasmodium parasites, which approximately $24 \mathrm{~h}$ after ingestion, penetrate the midgut epithelium to establish an infection on the basal sub-epithelial space that is an extension of the haemocoel. On the other hand, $A n$. coluzzii exhibits a low level of tolerance to gut microbiota, which in turn leads to low haemolymph

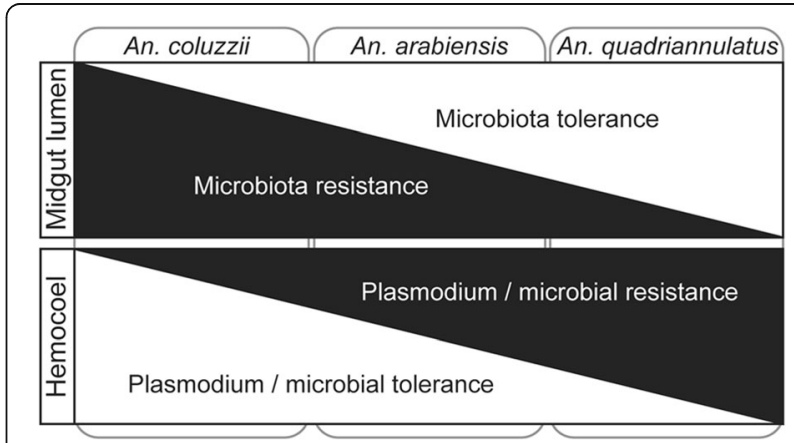

Fig. 4 Model of immune tolerance and resistance equilibrium. The model depicts two immune compartments, the midgut lumen and hemocoel, separated by the midgut epithelium. A highly efficient malaria vector, An. coluzzii (Ac), and non-vector An. quadriannulatus $(A q)$ are placed at different areas of the model by the observed interplay between the tolerance and resistance in the two immune compartments 
antimicrobial activity and increased tolerance to Plasmodium invasion. Intriguingly, the haemolymph antibacterial activity in An. coluzzii reduced drastically following a blood meal. This immune suppression may be part of the energy trade-off between the two immune compartments, whereby increased resistance to bacterial proliferation in the midgut after a blood meal is linked to immune repression and increased tolerance in the haemolymph. Indeed, it has been suggested that susceptibility to Plasmodium infection in An. gambiae is a result of purposeful immune suppression to prevent over-activation of immune response following ingestion of a blood meal [21, 48]. An example of such a response is the formation of ImPer-DUOX, a protein network that creates a barrier to restrict midgut-derived microbial immune elicitors from reaching immune receptors on epithelial cells. An. arabiensis appears to show moderate tolerance and resistance levels in both compartments.

Excessive or uncontrolled immune responses can have an adverse impact on fitness and reproduction. Female mosquitoes require a blood meal to obtain the necessary nutrients and energy for reproduction, but this is associated with increased risk of infection from blood-borne pathogens or their microbiota. The tolerance versus resistance model that we have proposed for the three mosquito species may represent different configurations of a resource management strategy that is in conjunction with accommodation of parasitism with Plasmodium. The malaria non-vector, An. quadriannulatus, tolerates an increase in bacteria after a blood meal but upregulates immune response in the haemocoel in anticipation of potential pathogen invasion, thus restricting Plasmodium infection. Conversely, the malaria vector, $A n$. coluzzii, has evolved to resist bacterial over-proliferation in the gut lumen and therefore limits haemolymph responses, perhaps as a result of its co-evolution and coadaptation with Plasmodium. The strategy of An. coluz$z i i$ appears to be more cost effective in the absence of Plasmodium infection, as demonstrated by the fact that this mosquito was shown to live longer. However, in the presence of infection, its survival is compromised, possibly due to gut bacteria that take advantage of epithelial invasion by Plasmodium to infect the non-immune haemolymph [49].

Beyond their significance in understanding immune response management and homoeostasis in mosquitoes, our data reveal new opportunities for exploring potential targets for developing novel vector and malaria control tools. Targeting tolerance or resistance to microbiota in the mosquito gut could be exploited towards modifying the mosquito immune response against Plasmodium in the haemocoel and inducing premature death due to the collateral damage of internal organs. It is plausible to achieve total Plasmodium transmission blockade using transgenic mosquito approaches. Using this approach, the mosquito immune system can be designed to augment parasite killing by targeting mosquito anti-malarial regulators that function upon binding to the parasite surface, for example, SPCLIP1 that binds to the ookinete and facilitates complement attacks [5]. Alternatively, the premature killing of mosquito vectors, before the parasite completes the gonotrophic cycle, can be achieved through transgenic approaches. This includes the constitutive knockout or overexpression of bacterial receptors that disrupt gut homeostasis and eventually kill the mosquito due to opportunistic infections or altered physiology. Some receptors, with a significant role as regulators of gut antibacterial defences, have been identified. These include PGRPLC/A, fibronectin type-III domain proteins (FN3D1-3) and a gustatory G-protein coupled receptor (GPRGR9), which can be exploited to reduce mosquito longevity.

\section{Conclusion}

The level of immune response (tolerance/resistance) in the midgut, often against microbiota, appears to regulate the level of immune response in the haemolymph, possibly due to priming of complement reactions. Malaria non-vector mosquito species, An. quadriannulatus, show a higher tolerance to gut microbiota, resulting in a greater refractoriness to Plasmodium infection, while the opposite is true for malaria vector mosquitoes. An indepth understanding of the molecular mechanisms regulating immune tolerance versus resistance in different mosquito vectors of malaria could guide the design of new vector and disease control strategies.

\section{Additional file}

Additional file 1: Table S1. Oligonucleotide primers used in gene abundance measurements and silencing. (DOCX $16 \mathrm{~kb}$ )

\section{Abbreviations \\ APL1: Anopheles-Plasmodium-responsive leucine-rich repeat 1; CSP: Circumsporozoite protein; Duox-IMPer: Dual oxidase - immunomodulatory peroxidase; GFP: Green fluorescent protein; Imd: Immune deficiency pathway; NF-kB: Nuclear factor-kappaB; PGRP: Peptidoglycan recognition protein; qPCR: Quantitative real-time poly- merase chain reaction; RLIM1: Leucine-rich repeat proteins 1; RT- qPCR: Reverse-transcription qPCR; TEP1: Thioester-containing protein 1}

\section{Acknowledgments}

We thank Katarzyna Sala for technical support and Idir Akhouayri for critical reading of the manuscript.

\section{Funding}

The work was supported by grants from the Wellcome Trust, WT093587MA and the BBSRC Project BB/K009338/1.

Availability of data and material

The datasets and analysed data during the current study are available from the corresponding author on reasonable request. 


\section{Authors' contributions}

Conceived and designed the experiments: TH, GKC. Performed the experiments: TH, ZG. Analysed the data: TH, GKC. Contributed reagents/ materials/analysis tools: TH, GKC. Wrote the manuscript: TH, ZG and GKC. All authors read and approved the final manuscript.

\section{Competing interests}

The authors declare that they have no competing interests.

\section{Consent for publication}

Not applicable.

\section{Ethics approval and consent to participate}

All experiments involving animals were carried out in accordance with the United Kingdom Animals (Scientific Procedures) Act 1986. The protocol for infecting mosquitoes with $P$. berghei by blood-feeding on parasite-infected mice was approved and carried out under the UK Home Office License PPL70/7185. Animals were anaesthetised prior to mosquito feeding or blood drawing using a Rompun-Ketamine combination. Euthanasia was performed by cervical dislocation following administration of a high dose of anaesthesia. The numbers of animals used in these experiments were minimised by incorporation of the most economical protocols. Opportunities for reduction, refinement and replacement of animal experiments were constantly monitored.

\section{Publisher's Note}

Springer Nature remains neutral with regard to jurisdictional claims in published maps and institutional affiliations.

\section{Author details}

'Department of Life Sciences, Imperial College London, London, UK. 2Department of Comparative Physiology and Biometrics, University of Ghent, Ghent, Belgium. ${ }^{3}$ Costello Medical Consulting, Cambridge, UK

Received: 10 August 2016 Accepted: 24 March 2017

\section{Published online: 18 April 2017}

\section{References}

1. Dimopoulos G. Insect immunity and its implication in mosquito-malaria interactions. Cell Microbiol. 2003;5(1):3-14

2. Dong Y, Manfredini F, Dimopoulos G. Implication of the mosquito midgut microbiota in the defense against malaria parasites. PLoS Pathog. 2009;5(5): e1000423.

3. Gendrin M, Christophides GK. The Anopheles mosquito microbiota and their impact on pathogen transmission, Anopheles mosquitoes - New insights into malaria vectors, 2013. In: Manguin S, editor, InTech, doi:10.5772/55107.

4. Stathopoulos S, Neafsey DE, Lawniczak MK, Muskavitch MA, Christophides GK. Genetic dissection of Anopheles gambiae gut epithelial responses to Serratia marcescens. PLoS Pathog. 2014;10(3):e1003897.

5. Povelones M, Bhagavatula L, Yassine $H$, Tan LA, Upton LM, Osta MA, Christophides GK. The CLIP-domain serine protease homolog SPCLIP1 regulates complement recruitment to microbial surfaces in the malaria mosquito Anopheles gambiae. PLoS Pathog. 2013;9(9):e1003623.

6. Blandin S, Shiao SH, Moita LF, Janse CJ, Waters AP, Kafatos FC, Levashina EA. Complement-like protein TEP1 is a determinant of vectorial capacity in the malaria vector Anopheles gambiae. Cell. 2004;116(5):661-70

7. Dong Y, Aguilar R, Xi Z, Warr E, Mongin E, Dimopoulos G. Anopheles gambiae immune responses to human and rodent Plasmodium parasite species. PLoS Pathog. 2006;2(6):e52.

8. Le BV, Williams M, Logarajah S, Baxter RH. Molecular basis for genetic resistance of Anopheles gambiae to Plasmodium: structural analysis of TEPT susceptible and resistant alleles. PLoS Pathog. 2012;8(10):e1002958.

9. Molina-Cruz A, DeJong RJ, Ortega C, Haile A, Abban E, Rodrigues J, et al. Some strains of Plasmodium falciparum, a human malaria parasite, evade the complement-like system of Anopheles gambiae mosquitoes. Proc Natl Acad Sci U S A. 2012;109(28):E1957-62.

10. Blandin SA, Wang-Sattler R, Lamacchia M, Gagneur J, Lycett G, Ning Y, et al. Dissecting the genetic basis of resistance to malaria parasites in Anopheles gambiae. Science. 2009;326(5949):147-50.

11. Kinnebrew MA, Pamer EG. Innate immune signaling in defense against intestinal microbes. Immunol Rev. 2012;245(1):113-31.
12. Christophides GK, Zdobnov E, Barillas-Mury C, Birney E, Blandin S, Blass C, et al. Immunity-related genes and gene families in Anopheles gambiae. Science. 2002;298(5591):159-65.

13. Frolet C, Thoma M, Blandin S, Hoffmann JA, Levashina EA. Boosting NF-kBdependent basal immunity of Anopheles gambiae aborts development of Plasmodium berghei. Immunity. 2006;25(4):677-85.

14. Li MW, Wang J, Zhao YO, Fikrig E. Innexin AGAP001476 is critical for mediating anti-Plasmodium responses in Anopheles mosquitoes. J Biol Chem. 2014:289(36):24885-97.

15. Schneider DS, Ayres JS. Two ways to survive infection: what resistance and tolerance can teach us about treating infectious diseases. Nat Rev Immunol. 2008;8(11):889-95.

16. Raberg L, Graham AL, Read AF. Decomposing health: tolerance and resistance to parasites in animals. Philos Trans R Soc Lond B Biol Sci. 2009;364(1513):37-49.

17. Medzhitov R, Schneider DS, Soares MP. Disease tolerance as a defense strategy. Science. 2012;335(6071):936-41.

18. Aggarwal K, Rus F, Vriesema-Magnuson C, Erturk-Hasdemir D, Paquette N, Silverman N. Rudra interrupts receptor signaling complexes to negatively regulate the IMD pathway. PLoS Pathog. 2008:4(8):e1000120.

19. Lhocine N, Ribeiro PS, Buchon N, Wepf A, Wilson R, Tenev T, et al. PIMS modulates immune tolerance by negatively regulating Drosophila innate immune signaling. Cell Host Microbe. 2008;4(2):147-58.

20. Zaidman-Remy A, Herve M, Poidevin M, Pili-Floury S, Kim MS, Blanot D, et al. The Drosophila amidase PGRP-LB modulates the immune response to bacterial infection. Immunity. 2006;24(4):463-73.

21. Kumar S, Molina-Cruz A, Gupta L, Rodrigues J, Barillas-Mury C. A peroxidase/ dual oxidase system modulates midgut epithelial immunity in Anopheles gambiae. Science. 2010;327(5973):1644-8.

22. Round JL, O'Connell RM, Mazmanian SK. Coordination of tolerogenic immune responses by the commensal microbiota. J Autoimmun. 2010;34(3):J220-5.

23. Rescigno M. Gut commensal flora: tolerance and homeostasis. F1000 Biol Rep. 2009;1:9.

24. Silipo A, Erbs G, Shinya T, Dow JM, Parrilli M, Lanzetta R, et al. Glycoconjugates as elicitors or suppressors of plant innate immunity. Glycobiology. 2010;20(4):406-19.

25. Aslam SN, Newman MA, Erbs G, Morrissey KL, Chinchilla D, Boller T, et al. Bacterial polysaccharides suppress induced innate immunity by calcium chelation. Curr Biol. 2008;18(14):1078-83.

26. Milling A, Babujee L, Allen C. Ralstonia solanacearum extracellular polysaccharide is a specific elicitor of defense responses in wilt-resistant tomato plants. PLoS One. 2011;6(1):e15853.

27. Muturi EJ, Mbogo CM, Mwangangi JM, Ng'ang'a ZW, Kabiru EW, Mwandawiro C, Beier JC. Concomitant infections of Plasmodium falciparum and Wuchereria bancrofti on the Kenyan coast. Filaria J. 2006;5:8.

28. Habtewold T, Povelones M, Blagborough AM, Christophides GK Transmission blocking immunity in the malaria non-vector mosquito Anopheles quadriannulatus species A. PLoS Pathog. 2008:4(5):e1000070.

29. Scott JA, Brogdon WG, Collins FH. Identification of single specimens of the Anopheles gambiae complex by the polymerase chain reaction. Am J Trop Med Hyg. 1993;49(4):520-9.

30. Sinden RE, Dawes EJ, Alavi Y, Waldock J, Finney O, Mendoza J, et al. Progression of Plasmodium berghei through Anopheles stephensi is densitydependent. PLoS Pathog. 2007:3(12):e195.

31. Meister S, Agianian B, Turlure F, Relogio A, Morlais I, Kafatos FC, Christophides GK. Anopheles gambiae PGRPLC-mediated defense against bacteria modulates infections with malaria parasites. PLoS Pathog. 2009;5(8):e1000542.

32. Frank JA, Reich Cl, Sharma S, Weisbaum JS, Wilson BA, Olsen GJ. Critical evaluation of two primers commonly used for amplification of bacterial 165 rRNA genes. Appl Environ Microbiol. 2008;74(8):2461-70.

33. Hultmark D, Engstrom A, Bennich H, Kapur R, Boman HG. Insect immunity: isolation and structure of cecropin $D$ and four minor antibacterial components from Cecropia pupae. Eur J Biochem. 1982:127(1):207-17.

34. Dong Y, Dimopoulos G. Anopheles fibrinogen-related proteins provide expanded pattern recognition capacity against bacteria and malaria parasites. J Biol Chem. 2009;284(15):9835-44.

35. Gendrin M, Rodgers FH, Yerbanga RS, Ouédraogo JB, Basáñez M-G, Cohuet A Christophides GK. Antibiotics in ingested human blood affect the mosquito microbiota and capacity to transmit malaria. Nat Commun. 2015:6:5921.

36. Meister S, Kanzok SM, Zheng XL, Luna C, Li TR, Hoa NT, et al. Immune signaling pathways regulating bacterial and malaria parasite infection of the mosquito Anopheles gambiae. Proc Natl Acad Sci-Biol. 2005;102(32):11420-5. 
37. Takken W, Eling W, Hooghof J, Dekker T, Hunt R, Coetzee M. Susceptibility of Anopheles quadriannulatus Theobald (Diptera: Culicidae) to Plasmodium falciparum. Trans R Soc Trop Med Hyg. 1999;93(6):578-80.

38. Gneme A, Guelbeogo WM, Riehle MM, Sanou A, Traore A, Zongo S, et al. Equivalent susceptibility of Anopheles gambiae $\mathrm{M}$ and $\mathrm{S}$ molecular forms and Anopheles arabiensis to Plasmodium falciparum infection in Burkina Faso. Malar J. 2013;12:204.

39. Salvaudon L, Heraudet V, Shykoff JA. Genotype-specific interactions and the trade-off between host and parasite fitness. BMC Evol Biol. 2007;7:189.

40. Harris C, Morlais I, Churcher TS, Awono-Ambene P, Gouagna LC, Dabire RK, et al. Plasmodium falciparum produce lower infection intensities in local versus foreign Anopheles gambiae populations. PLoS One. 2012;7(1):e30849.

41. Povelones M, Upton LM, Sala KA, Christophides GK. Structure-function analysis of the Anopheles gambiae LRIM1/APL1C complex and its interaction with complement C3-like protein TEP1. PLoS Pathog. 2011;7(4):e1002023.

42. Mendes AM, Awono-Ambene PH, Nsango SE, Cohuet A, Fontenille D, Kafatos FC, et al. Infection intensity-dependent responses of Anopheles gambiae to the African malaria parasite Plasmodium falciparum. Infect Immun. 2011;79(11):4708-15.

43. Medley GF, Sinden RE, Fleck S, Billingsley PF, Tirawanchai N, Rodriguez MH. Heterogeneity in patterns of malarial oocyst infections in the mosquito vector. Parasitology. 1993;106(Pt 5):441-9.

44. Basanez MG, Townson H, Williams JR, Frontado H, Villamizar NJ, Anderson RM. Density-dependent processes in the transmission of human onchocerciasis: relationship between microfilarial intake and mortality of the simuliid vector. Parasitology. 1996:113(Pt 4):331-55

45. Kause A, van Dalen S, Bovenhuis H. Genetics of ascites resistance and tolerance in chicken: a random regression approach. G3 (Bethesda). 2012; 2(5):527-35.

46. Raberg $L$, Sim D, Read AF. Disentangling genetic variation for resistance and tolerance to infectious diseases in animals. Science. 2007;318(5851):812-4.

47. Xi Z, Ramirez JL, Dimopoulos G. The Aedes aegypti toll pathway controls dengue virus infection. PLoS Pathog. 2008;4(7):e1000098.

48. Cirimotich CM, Ramirez JL, Dimopoulos G. Native microbiota shape insect vector competence for human pathogens. Cell Host Microbe. 2011;10(4):307-10

49. Rodrigues J, Brayner FA, Alves LC, Dixit R, Barillas-Mury C. Hemocyte differentiation mediates innate immune memory in Anopheles gambiae mosquitoes. Science. 2010;329(5997):1353-5.

\section{Submit your next manuscript to BioMed Central} and we will help you at every step:

- We accept pre-submission inquiries

- Our selector tool helps you to find the most relevant journal

- We provide round the clock customer support

- Convenient online submission

- Thorough peer review

- Inclusion in PubMed and all major indexing services

- Maximum visibility for your research

Submit your manuscript at www biomedcentral.com/submit
() BioMed Central 